\title{
Hyperanalyticity of space-time
}

\author{
Aleksandr Rybnikov
}

08.21 .2018

\begin{abstract}
New mathematical object - hyperanalytic function is introduced. The convergence of hyperanalytic functions is substantially above than the convergence of analytic functions. A specific sample of hyperanalytic function is the reticulum function $(\mathrm{RF})$. This function describes the reticulum spacetime. RF can't be decomposed into the Fourier series and, therefore, RF does not provide the conservation of parity as the analytic functions do. Thanks to this, the RF can be decomposed in an endless series of two primitive hyperanalytic functions by sequential attempts of decomposition in the even and odd functions. The unique parameter of such series is the fine structure constant $\alpha$. It allows combine all fundamental interactions into the Naturally-Unified Quantum Theory of Interactions. The price of such quantum unification is the reticulum space-time.
\end{abstract}

Keywords: fine structure constant $\alpha$, theory of everything, parity nonconservation. 


\section{Contents}

$\begin{array}{llr}1 & \text { Introduction } & 3\end{array}$

2 Decomposition of $\mathbf{R F} \quad \mathbf{5}$

2.1 The mean value of $\mathrm{RF} \ldots \ldots \ldots \ldots \ldots$

2.2 First difference . . . . . . . . . . . . . . . 5

2.3 Even differences . . . . . . . . . . . . . . . . . 6

2.4 Odd differences . . . . . . . . . . . . . . . . . . . . . . 7

2.5 Three-dimensional $\mathrm{RF} \ldots \ldots \ldots \ldots$

3 Quantum derivative with respect to time 10

4 Unified Theory of Interactions 12

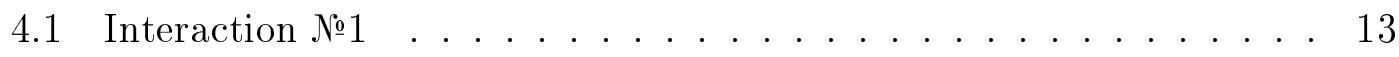

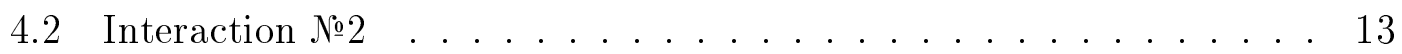

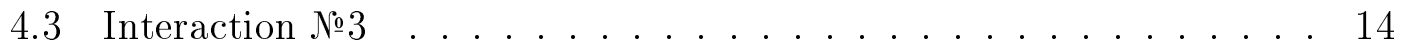

4.4 Interaction №4 f . . . . . . . . . . . . . . . . . . . . . 14

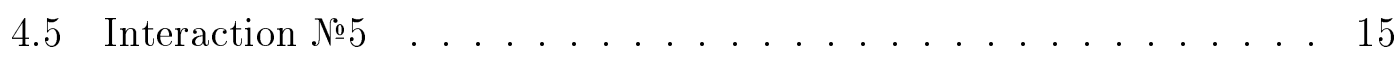

4.6 Interaction $\mathrm{No6} \ldots \ldots \ldots \ldots \ldots$

$\begin{array}{lll}5 & \text { Appendix } & 18\end{array}$ 


\section{Introduction}

It is known that there is a fundamental connection between analyticity of the function and the convergence of its Fourier coefficients. The better the function, the faster its coefficients tend to zero, and vice versa. The power decrease of Fourier coefficients is inherent in functions of the $C^{k}$ class while exponential to analytical functions. Here there is a possibility of existence of the hyperanalytic functions, for which the decrease of the Fourier coefficients corresponds to tetration ${ }^{[1]}$.

Examples of such functions were first obtained with conventional arithmetic ${ }^{[2]}$. Therefore for this article calculations have been made with a program that provides the necessary accuracy in each case $^{1}$.

Natural hyperanalytic function occurs when considering reticulum with a step $\mathrm{L}$, in which nodes there are not defined yet objects. The distribution of center's objects can be described using the reticulum functions (RF). The definition of a one-dimensional RF is based on the following identity ${ }^{2}$.

$$
\frac{1}{\sigma \sqrt{2 \pi}} \int_{-\infty}^{\infty} e^{-\frac{1}{2}\left(\frac{x}{\sigma}\right)^{2}} d x=\frac{1}{\sigma \sqrt{2 \pi}} \int_{-\frac{L}{2}}^{\frac{L}{2}} \sum_{n=-\infty}^{\infty} e^{-\frac{1}{2}\left(\frac{x-n L}{\sigma}\right)^{2}} d x=1 .
$$

From here $\mathrm{RF}^{3}$ is

$$
\mathbb{R}(x)=\frac{1}{\sigma \sqrt{2 \pi}} \sum_{n=-\infty}^{\infty} e^{-\frac{1}{2}\left(\frac{x-n L}{\sigma}\right)^{2}} .
$$

Graph 1. Hyperanalytic function $\mathbb{R}(x)^{4}$.

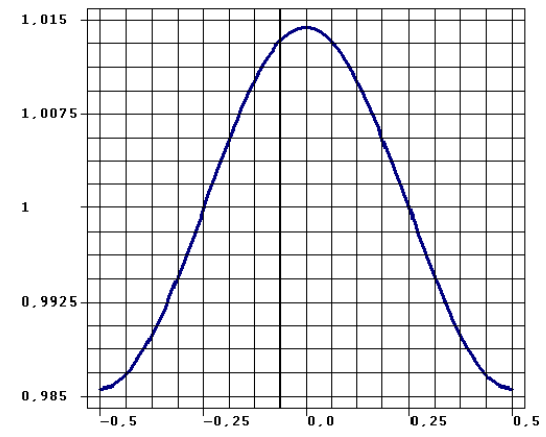

\footnotetext{
${ }^{1}$ The long arithmetic programs in the application were set up for calculations using more than 100 significant digits.

${ }^{2}$ For the first time the value of this one-dimensional integral was calculated in 1729 year by Leonard Euler during his work at the St. Petersburg Academy Of Sciences. Karl Friedrich Gauss, whose name is called the subintegral function of the first integral is born on April 30, 1977.

${ }^{3}$ The MATHEMATICAL DOUBLE-STRUCK CAPITAL font is used to highlight all hyperanalytic functions and constants produced from them.

${ }^{4}$ All calculations were performed at the values $L=1$ and $\sigma=0.4992619105929628$
} 
It is obvious that the RF can not be laid out in the Fourier series because it does not have antiderivative that can be expressed as elementary functions. By virtue of this RF cannot be decomposed into even and odd functions ${ }^{5}$, while an arbitrary analytic function $f$ can be only presented in the form of sum of odd and even functions in the interval $[a, b]$ :

$$
f(x)=g(x)+h(x),
$$

where

$$
\begin{aligned}
& g(x)=\frac{f(x-a)-f(b-x)}{2}, \\
& h(x)=\frac{f(x-a)+f(b-x)}{2} .
\end{aligned}
$$

Due to this the RF can be laid out in an endless row of two primitive hyperanalytic functions by sequential attempts to decompose on even and odd functions. Thus, the RF can be decomposed by the simplest way, but such a series is not one like the orthonormal basis of Fourier series.

\footnotetext{
${ }^{5}$ The absence of a certain parity is the non-preservation of parity.
} 


\section{Decomposition of RF}

\subsection{The mean value of $\mathrm{RF}$}

As it follows from (1.1) the mean value of RT is 1 . However as will be seen from the further, it is expedient to choose the greater value of the decomposition's constant member. Introduce the following definitions:

$$
\begin{gathered}
\mathbb{R}(0)=\mathbb{R}_{\max }=\frac{1}{\sigma \sqrt{2 \pi}} \sum_{n=-\infty}^{\infty} e^{-\frac{1}{2}\left(\frac{-n}{\sigma}\right)^{2}}, \\
\mathbb{R}(1 / 2)=\mathbb{R}_{\text {min }}=\frac{1}{\sigma \sqrt{2 \pi}} \sum_{n=-\infty}^{\infty} e^{-\frac{1}{2}\left(\frac{1 / 2-n}{\sigma}\right)^{2}} .
\end{gathered}
$$

Then $A_{0}$ is the mean value of $\mathrm{RF}$ :

$$
A_{0}=\frac{\mathbb{R}_{\max }+\mathbb{R}_{\min }}{2} .
$$

Graph 2. First difference $\mathbb{R}(x)-A_{0}$

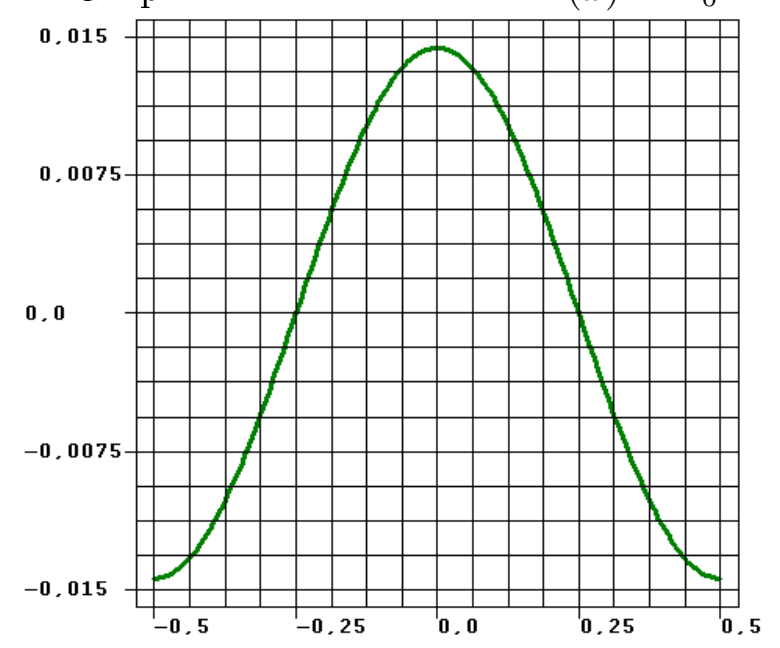

\subsection{First difference}

One can approximate first difference by the following way:

$$
A_{1}(x)=\frac{\mathbb{R}_{\max }-\mathbb{R}_{\min }}{2} \cos (2 \pi x) .
$$

Let introduce parameter of the fine structure $\alpha$ as function of $\sigma$ :

$$
\alpha(\sigma)=\frac{1}{2} \frac{\mathbb{R}_{\max }-\mathbb{R}_{\min }}{\mathbb{R}_{\max }+\mathbb{R}_{\min }}
$$


Now $A_{1}(x)$ can be expressed:

$$
A_{1}(x)=\frac{\mathbb{R}_{\max }+\mathbb{R}_{\min }}{2}(2 \alpha(\sigma) \cos (2 \pi x)) .
$$

The choice of the name and symbol of this parameter is due to the fact that

$$
\alpha(0.4992619105929628)=\alpha=\frac{e^{2}}{4 \pi \epsilon_{0} \hbar c}
$$

is the value known in physics as a fine structure constant ${ }^{[3]}$.

\subsection{Even differences}

Even differences are a primitive hyperanalytic function $\overline{\mathbb{V}}(2 i \times 2 \pi x)$, which is quasisymmetric relative to the point $\mathrm{x}=0.25$.

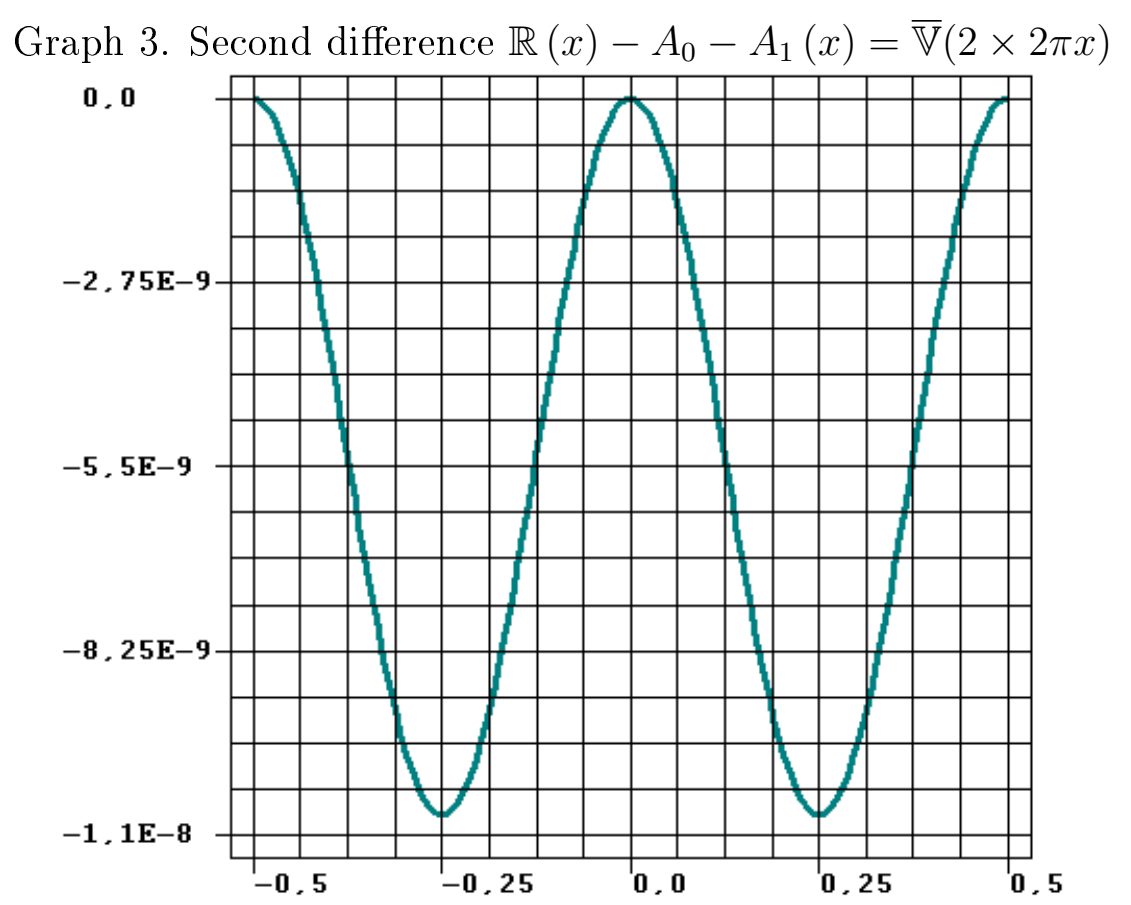

Its symmetrical part approximated in the following way:

$$
A_{2 i}(x)=c_{2 i}(\cos (2 i \times 2 \pi x)-1) .
$$

причём

$$
\sum_{i=1}^{\infty} c_{2 i}=\frac{\mathbb{R}_{\max }+\mathbb{R}_{\min }}{2}-1=2 * \sum_{k=1}^{\infty} \alpha^{4^{k}}
$$


Using the value

$$
\mathbb{R}(1 / 4)=\mathbb{R}_{1 / 4}=\frac{1}{\sigma \sqrt{2 \pi}} \sum_{n=-\infty}^{\infty} e^{-\frac{1}{2}\left(\frac{1 / 4-n}{\sigma}\right)^{2}}
$$

define the amplitude for $c_{2}: \frac{1}{2}\left(\frac{\mathbb{R}_{\max }+\mathbb{R}_{\min }}{2}-\mathbb{R}_{1 / 4}\right)=2 \alpha^{4}$. This definition allows to select approximation $A(x)$ in the form:

$$
A(x)=\frac{\mathbb{R}_{\max }+\mathbb{R}_{\min }}{2}(1+2 \alpha(\sigma) \cos (2 \pi x))+2 \alpha^{4}(\cos (2 \times 2 \pi x)-1) .
$$

\subsection{Odd differences}

Odd differences are a primitive hyperanalytic function $\mathbb{W}((2 i-1) \times 2 \pi x)$, which is quasiantisymmetric relative to the point $\mathrm{x}=0.25$.

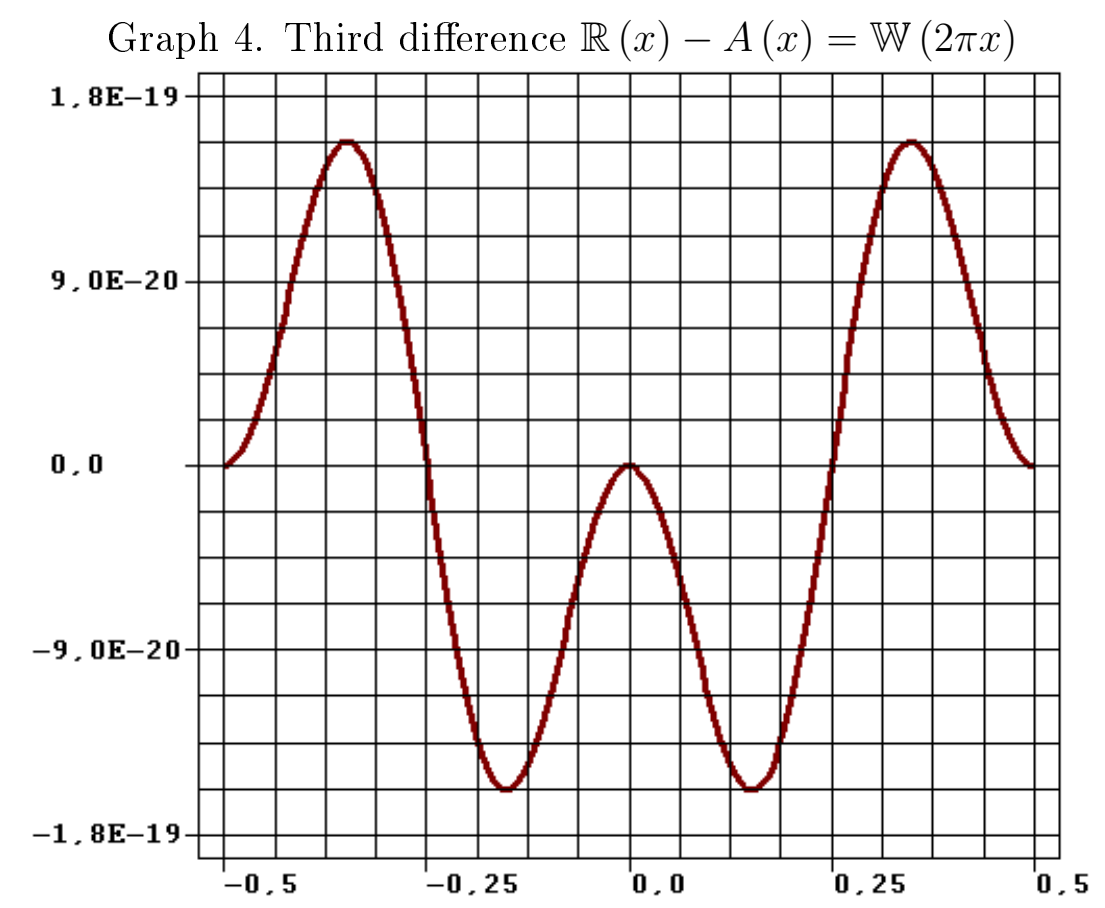

Quasiantisymmetry of $\mathbb{W}(2 \pi x)$ follows from the fact that the integral of $A(x)$ differs from 1 :

$$
\int_{-1 / 2}^{1 / 2} \mathrm{~A}(x) \mathrm{dx}-1=\frac{1}{4}\left(\mathbb{R}_{\max }+\mathbb{R}_{\min }\right)+\frac{1}{2} \mathbb{R}_{1 / 4}-1 \simeq 1.02 E-34 .
$$

Thus function $\mathbb{W}((2 i-1) \times 2 \pi x)$ should be decomposed in the even and odd function. Its even part is:

$\mathbb{W}^{\text {qs }}((2 i-1) \times 2 \pi x)=\frac{\mathbb{W}((2 i-1) \times 2 \pi x)+\mathbb{W}((2 i-1) \times 2 \pi(0.5-x))}{2}=\overline{\mathbb{V}}(2(i+1) \times 2 \pi x)$. 
This is seen from the following picture. However, as shown above, $\overline{\mathbb{V}}(2 i \times 2 \pi x)$ is not an even function.

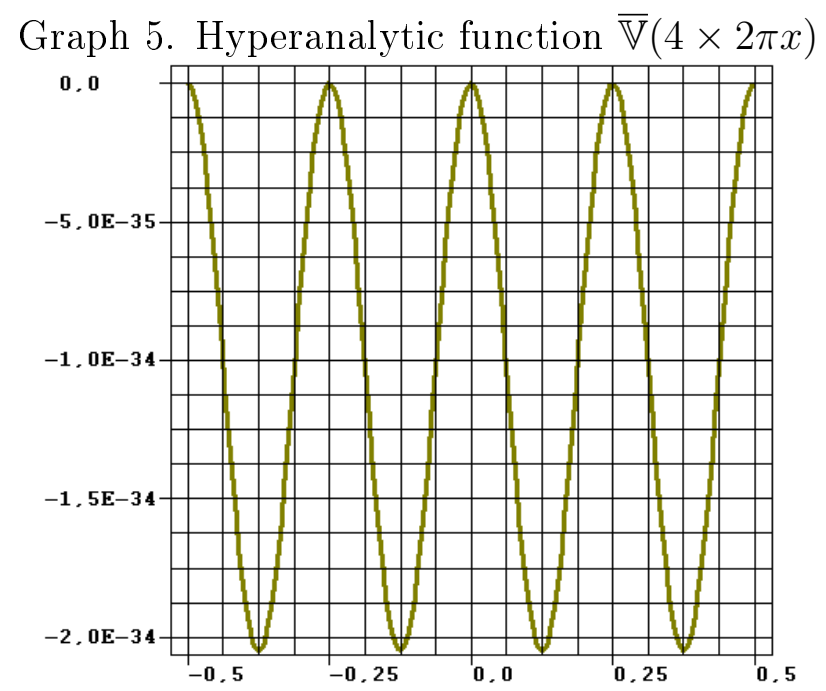

Graph 6. Hyperanalytic function $\mathbb{W}(3 \times 2 \pi x)$

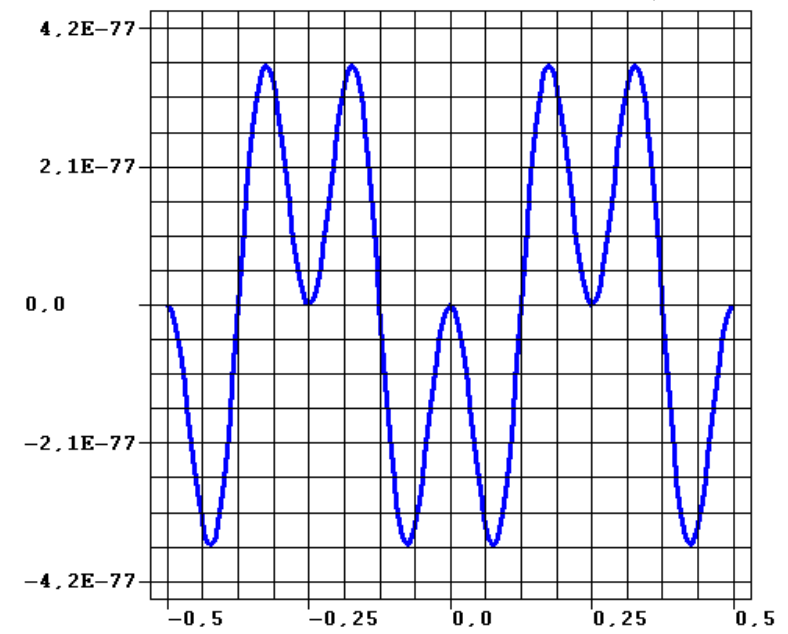

The odd part of $\mathbb{W}((2 i-1) \times 2 \pi x)$ is no longer a hyperanalytic function and is equal to:

$$
W^{\mathrm{qa}}((2 i-1) \times 2 \pi x)=\frac{\mathbb{W}((2 i-1) \times 2 \pi x)-\mathbb{W}((2 i-1) \times 2 \pi(0.5-x))}{2} .
$$

It can be approximated with any degree of accuracy following way:

$$
A\left(W^{\mathrm{qa}}((2 i-1) \times 2 \pi x)\right)=\beta(\cos (3(2 i-1) \times 2 \pi x)-\cos ((2 i-1) \times 2 \pi x)),
$$

where $\beta$ is a normalizing multiplier. 
Thus, the approximation of $\mathbb{R}(x)$ is:

$$
\begin{aligned}
A(x)=\frac{\mathbb{R}_{\text {max }}+\mathbb{R}_{\text {min }}}{2}(1 & +2 \alpha \cos (2 \pi x)) \\
& +2 \sum_{i=1}^{\infty} \alpha^{4^{i}}(\cos (2 i \times 2 \pi x)-1) \\
& +\frac{2}{\mathbb{W}_{\max }} \sum_{i=1}^{\infty} \alpha^{9 i^{2}}(\cos (3 \times(2 i-1) \times 2 \pi x)-\cos ((2 i-1) \times 2 \pi x)),
\end{aligned}
$$

where $\mathbb{W}_{\text {max }}$ is a normalizing multiplier.

\subsection{Three-dimensional RF}

Threed-imensional $\mathrm{RF} \mathbb{R}(x, y, z)$ can be obtained from the definition (1.2):

$$
\mathbb{R}(x, y, z)=\mathbb{R}_{\max }^{2} \mathbb{R}(x) .
$$

Thus, the approximation of the three-dimensional RF is also the series of the fine structure constant $\alpha$ along any axis of the reticulum three-dimensional space, and the constant itself is a function of the dimensionless parameter $\sigma$, which is equal to quotient of the "diameter" of some physical object, located in each cell, to the grid step L. 


\section{Quantum derivative with respect to time}

To quantize the time the direct use of the lattice idea is too formal. It is therefore appropriate to use a definition of derivative with respect to time but without moving to the limit. Let $\mathbb{R}(t)$ is $\mathrm{RF}$ on a unit interval $[-\mathrm{T} / 2, \mathrm{~T} / 2]$ and $\tau=\sigma$ и $\mathrm{T}=1$ :

$\mathbb{R}(t)=\frac{1}{\tau \sqrt{2 \pi}} \sum_{i=-\infty}^{\infty}\left[\exp \left(-\frac{1}{2}\left(\frac{t+\mathrm{T} / 4-i}{\tau}\right)^{2}\right)-\exp \left(-\frac{1}{2}\left(\frac{t-\mathrm{T} / 4-i}{\tau}\right)^{2}\right)\right]$.

Graph 7. Hyperanalytic function $\mathbb{R}(t)$

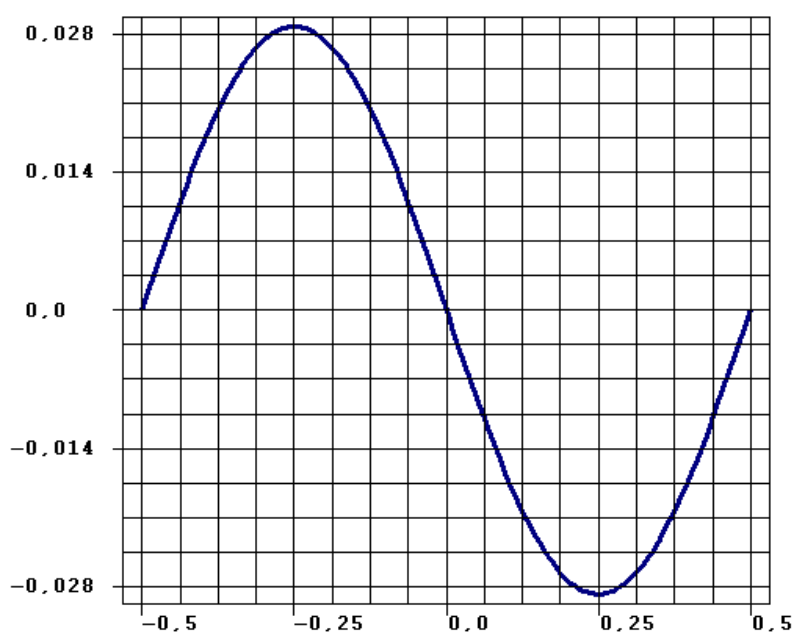

By consistently subtracting sinuses, one can show that the approximation of the $\mathbb{R}(t)$ has the following form:

$$
A(t)=\sum_{k=0}^{\infty}(-1)^{k+1} a_{k} \sin (2 \pi(2 k+1) t) .
$$

Let use $k+1$ equations with different values of $l$ to determine the coefficient's values $a_{k}$ :

$$
\sum_{i=0}^{k}(-1)^{i} a_{i} \sin \left(\frac{2 i+1}{2 l+1} \frac{2 \pi}{4}\right)=\mathbb{R}\left(\frac{1}{4(2 l+1)}\right) .
$$

Given that $A(1 / 4)$ is numerically equal to $2\left(\mathbb{R}_{\max }(\tau)+\mathbb{R}_{\min }(\tau)\right) \alpha(\tau)$, equation (5) can be written as follows:

$$
\alpha_{e f f}(t, \tau)=\frac{1}{2\left(\mathbb{R}_{\max }(\tau)+\mathbb{R}_{\min }(\tau)\right)} \sum_{k=0}^{\infty}(-1)^{k+1} a_{k} \sin (2 \pi(2 k+1) t) .
$$


$\mathbb{R}(t)$ is also a hyperanalytic function, as the next approximation takes place:

$$
\alpha_{e f f}(t, \tau)=\sum_{k=0}^{\infty}(-1)^{k+1} \alpha^{(2 k+1)^{2}} \sin (2 \pi(2 k+1) t) .
$$

Graph 8. Second harmonic.

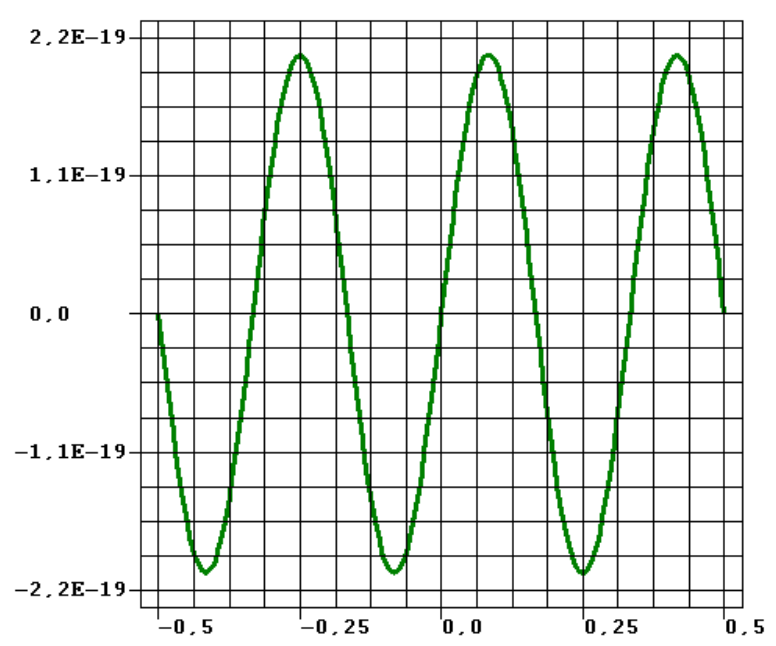

Graph 9. Third harmonic.

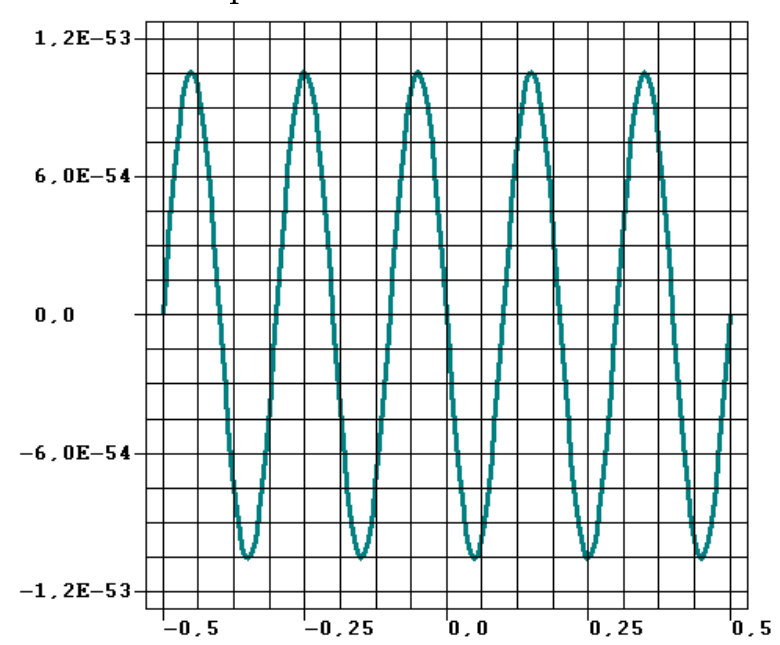




\section{Unified Theory of Interactions}

The resulting decomposition of hyperanalytic functions by the degrees of $\alpha$ allows to assert that a Naturally-Unified Quantum Theory of Interactions exists, because the information that can be extracted from the function $e^{-\frac{1}{2}\left(\frac{x}{\sigma}\right)^{2}}$ (or more precisely from formulas (2) and (5)) is at least equivalent to that contained in given below figure.

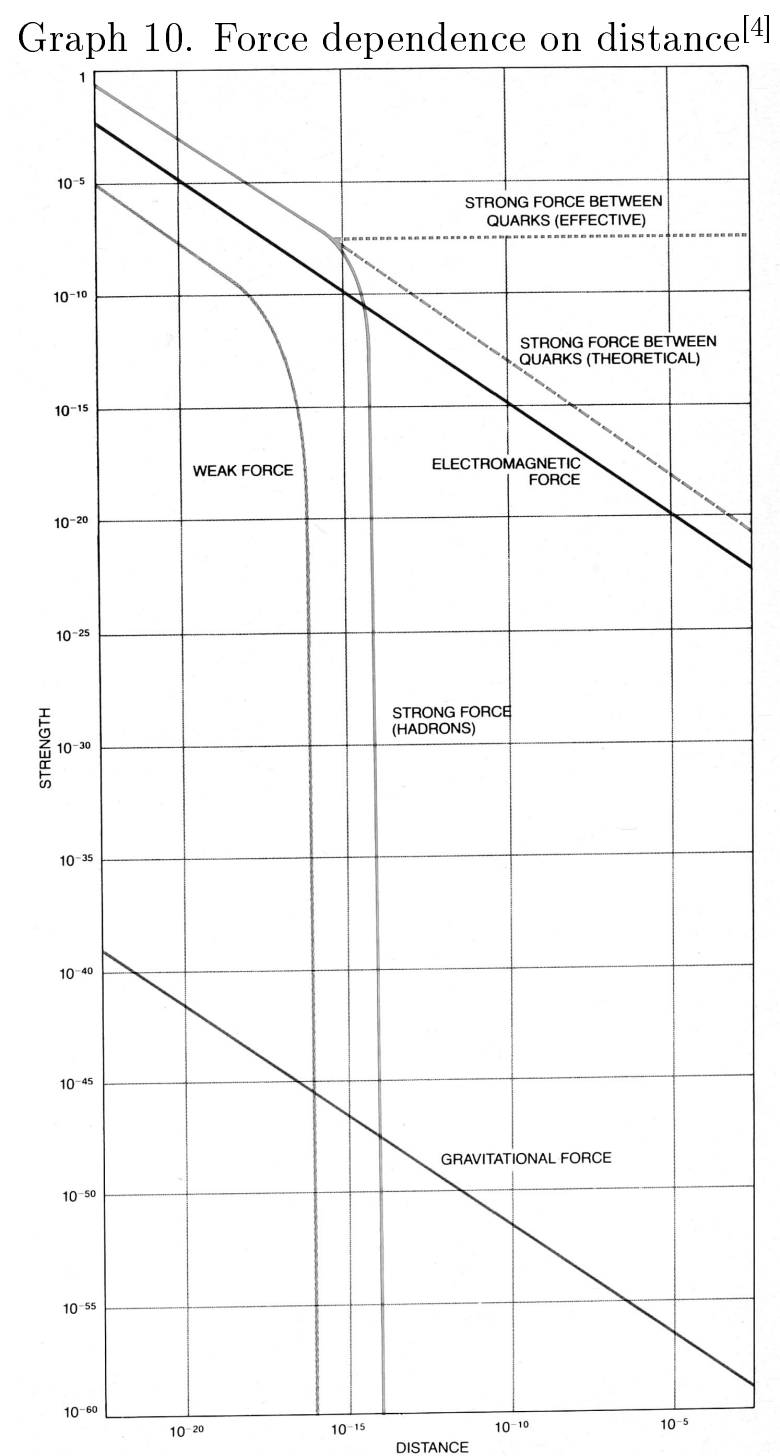

The key to decoding this information is the reticulum model of Space-Time. Independence of the obtained results from the size of $\mathrm{L}$ and $\mathrm{T}$ is therefore funda- 
mentally important ${ }^{6}$. This means that when considering the each interaction is due to a reticulum with specific values ${ }^{7}$. The reticulum Space-Time model allows to highlight four interactions from the decomposition of the RF:

- strong magnetic interaction (№1),

- electromagnetic interaction (№2),

- interference electro-weak interaction (№3),

- weak interaction (№4),

and three interactions from the decomposition of the quantum derivative with respect to time:

- electromagnetic interaction (№2),

- gravitational interaction (№5),

- unknown interaction (№6).

\subsection{Interaction №1}

The mean value of RT is equal 1 eventually. It is therefore advisable consider its value relative to the coefficient of the second member. In this case, it will have a known physical value ${ }^{[6]}$ :

$$
\frac{q_{S}}{q_{e}}=\frac{q_{N}}{q_{p}}=\frac{1}{2 \alpha}
$$

where $q_{S}$ and $q_{N}$ - are the Dirac monopole's magnetic charges, $q_{e}$ is the charge of electron, $q_{p}$ is the charge of positron. It means that the reticulum space is formed by the Dirac monopole's. Such a model was described in article $\mathrm{e}^{[2]}$.

\subsection{Interaction №2}

Coefficient at $\cos (2 \pi x)$ is equal $2 \alpha$. Assuming that the deuce reflects the existence of two photon states, it can't be taken into account when estimating intensity. In this way, as it should be to expect the intensity of electromagnetic interaction is $\alpha$.

\footnotetext{
${ }^{6}$ This is due to the fact that the normal distribution is infinitely divisible distribution.

${ }^{7}$ The experimental confirmation of this demonstrated the result of work ${ }^{[5]}$, that the optical transparency of the monoatomic 2M-layer of graphene depends only on the dimensionless values: constant fine structure $\alpha$ and number $\pi$.
} 


\subsection{Interaction №3}

As seen in Fig. 10 dependence of the magnitude of the weak forces on the distance has significantly different areas. At the starting site this dependence parallel to the dependence of electromagnetic forces. Odd differences contain $\cos (2 i \times 2 \pi x)$ with a double argument that corresponds to the description of interference interaction of photons and neutrino. Coefficient at $\cos (2 \times 2 \pi x)$ is equal $2 \alpha^{4}$. Assuming that the deuce reflects the existence of two photon and neutrino states, it can't be taken into account when estimating intensity. Because frequency increase (compared to the previous cosine), the value of the intensity of interference of the weak interaction has value $\sqrt{2} \alpha^{4}=4.01 \times 10^{-9}$. This value corresponds to end of a straight section. Odd difference with other values coefficients and other frequencies are repeated regularly for subsequent generations of leptons.

The importance of identifying this interaction is unique property of the primitive hyperanalytic function $\overline{\mathbb{V}}(2 i \times 2 \pi x)$ - not saving parity.

\subsection{Interaction №4}

The actual weak interaction corresponds to the $\cos (3 \times 2 \pi x)$ and $\cos (2 \pi x)$ with coefficient $2 \alpha^{9}$. Assuming that the deuce reflects the existence of two neutrino states, it can't be taken into account when estimating intensity. In view of the increasing frequency in three times the value of the intensity of the interference interaction should be multiplied by $\sqrt{3}$. Due to the function $\mathbb{W}((2 i-1) \times 2 \pi x)$ is not normalizied the coefficient should be divided by its maximum value $\mathbb{W}_{\text {max }}$ equal to $\cong 1.5396$. As a result get the value $\sqrt{3} \alpha^{9} / \mathbb{W}_{\max }=6.60 \times 10^{-20}$. This value corresponds to the end of the curved section. Odd differences with other values of coefficients and other frequencies are repeated regularly for subsequent generations of leptons, overlapping the entire range of together with the interaction of № 3 .

The importance of identifying this interaction is a unique property of a primitive hyperanalytic function $\mathbb{W}((2 i-1) \times 2 \pi x)$ - the nonconservation of parity.

Interactions of № 3 and № 4 are related to each other by a set of two unorthogonal hyperanalytic functions. This means that interacting leptons described initially as mixed states, regardless of whether the neutrino are massless or not. Thus, the transformations of neutrino one generations in the neutrino of another generation are natural quantum phenomenon.

As seen in Fig. 10 dependence of the magnitude of the weak forces on the distance has a plot at which the speed of their reduction is described precisely by coefficients of decomposition of the RF. The values of the lower bounds given in the following table show that the leptons of the fourth generations cannot exist because interactions № 3 and № 4 overlap each other eventually in this range due 
to the different speeds of reducing the lower bounds of each interaction.

\begin{tabular}{|c|c|c|}
\hline Generation & Interaction №3 & Interaction №4 \\
\hline \hline 1 & $\sqrt{2} \alpha^{4}$ & $\sqrt{3} \alpha^{9} / \mathbb{W}_{\max }$ \\
\hline 2 & $\sqrt{4} \alpha^{16}$ & $\sqrt{6} \alpha^{36} / \mathbb{W}_{\max }$ \\
\hline 3 & $\sqrt{8} \alpha^{64}$ & $\sqrt{9} \alpha^{81} / \mathbb{W}_{\max }$ \\
\hline
\end{tabular}

\subsection{Interaction №5}

Since the first coefficient of decomposition of the quantum derivative with respect to time has already identified as the intensity of electromagnetic interaction, it can be expected that the second factor is related to the only remaining interaction - gravitational. To get the intensity of gravitational interaction ${ }^{[4]}$ it is enough to square second coefficient $\alpha^{9}$ and multiply it by $\sqrt{3}$ (to account for another frequency).

The resulting value is less than a percentage exceeds the constant of gravitational interaction:

$$
G \frac{m_{p}^{2}}{\hbar c}=5.906 \times 10^{-38}
$$

This discrepancy gives an upper estimate of the quantum amendment, which can be added to the law of gravity.

First we show how the constant $G$ will look like if instead of the mass proton $m_{p}$ enter a new associated mass of proton $m_{p a}$. In this case, the value of $G$ will be the following type ${ }^{8}$ :

$$
G=\sqrt{3} \alpha^{18} \frac{\hbar c}{m_{p a}^{2}} \cdot
$$

Based on data ${ }^{10}$ in the following table, we obtain:

$$
m_{p a}=1.68082 \times 10^{-27} .
$$

${ }^{8}$ The obtained formula reveals the latent quantum-relativistic status of the law of gravity itself. The thing is that the product $\hbar \times c$ can be saved only under the simultaneous transformations $c$ $\rightarrow \infty$ and $\hbar \rightarrow 0$ according to the correspondence principle. Thus, to speak about the unilateral refinement of the Newton's Law of gravitation turns out to be wrong in principle.

${ }^{9}$ In 1922 , the Chicago physicist Arthur Lund suggested ${ }^{[7]}$ that the fine structure constant was somehow related to the nuclear mass defect, and also considered its possible relationship with gravity through the ratio:

$$
\frac{G m_{e}^{2}}{e^{2}}=\frac{\alpha^{17}}{2048 \pi^{6}},
$$

where $e$ и $m_{e}$ - charge and mass of electron.

${ }^{10}$ Data taken from Wikipedia 07.03.2018. 
Thus, the value of $m_{p a}$ by 9 electronic masses exceeds mass of the proton $m_{p}$ and can be considered reliable ${ }^{11}$.

\begin{tabular}{|c|c|}
\hline Constant & Value \\
\hline \hline$\hbar$ & $1.054571800(13) \times 10^{-34}$ Дж $\cdot \mathrm{c}$ \\
\hline $\mathrm{c}$ & $299792458 \mathrm{M} / \mathrm{c}$ \\
\hline$\alpha$ & $7.2973525664(17) \times 10^{-3}$ \\
\hline $\mathrm{G}$ & $6.67408(31) \times 10^{-11} \mathrm{~m}^{3} \cdot \mathrm{c}^{-2} \cdot \mathrm{K \Gamma}^{-1}$ \\
\hline
\end{tabular}

As an example of an estimate of $m_{p a}$, one can assume that this value includes the mass of the proton $m_{p}$ and the mass of the electron $m_{e}$. Besides, it is necessary to include mass of neutron $m_{n}$ with coefficient delta - a fraction of neutrons per proton, which is the tenth for stars and units for planets. It is also necessary to subtract the energy of the bound nucleons, which is different for stars and planets. Finally, we need to add kinetic energy to nucleon and other possible deposits. As a result the constant $G$ will become a constant $G_{i k}$, where, for example, $i$ and $k$ indices of the sun and planets.

\subsection{Interactions №6 and higher}

The remaining members of the decomposition of the discrete derivative of the RF can be interpreted only as interactions are essentially weaker than gravitational.

\footnotetext{
${ }^{11}$ It is more correct to say that now the accuracy of $m_{p a}$ is determined by accuracy $G$, not vice versa.
} 


\section{References}

[1] Tetration - Wikipedia

[2] Aleksandr Rybnikov, Space-time from the Gaussian function's point of view, http://www.gaussianfunction.com

[3] Coupling constant - Wikipedia

[4] 'T H o o f t G. Gauge Theories of the Forces between Elementary Particles.Scientific American, June 1980, v. 242, pp. 90 - 116.

[5] R. R. Nair, P. Blake, A. N. Grigorenko, K. S. Novoselov, T. J. Booth, T. Stauber, N. M. R. Peres, A. K. Geim. Fine Structure Constant Defines Visual Transparency of Graphene. Science 320, 1308 (2008) DOI:10.1126/science.1156965

[6] P.A.M. Dirac, Quantized Singularities in the Electromagnetic Field, Proceedings of the Royal Society, A133 (1931) pp 60-72.

[7] A. C. Lunn. Atomic Constants and Dimensional Invariants // Physical Review. - 1922. - Vol. 20. - P. 1-14. 


\section{$5 \quad$ Appendix}

The program works with numbers that are represented by strings with fixed length (Mlen). For carrying out the presented in the article computations are enough numbers in the range $(-99,+999)$. Negative the numbers start at 9 . The decimal point is not included, but it is assumed that it could be between the third and the fourth position. Constants have the following values:

Const $\mathrm{c} 4$ As Integer $=4$

Const c5 As Integer $=5$

Const c6 As Integer $=6$

Const $\mathrm{c} 10$ As Integer $=10$

Const $\mathrm{c} 47$ As Integer $=47$

Const c48 As Integer $=48$

Const $\mathrm{c} 49$ As Integer $=49$

Const $\mathrm{c} 96$ As Integer $=\mathrm{c} 48+\mathrm{c} 48$

Const c95 As Integer $=\mathrm{c} 96-1$

Const Zs As String * MLen = "0...0"

The following are the programs for four arithmetic operations and operation of sign's change.

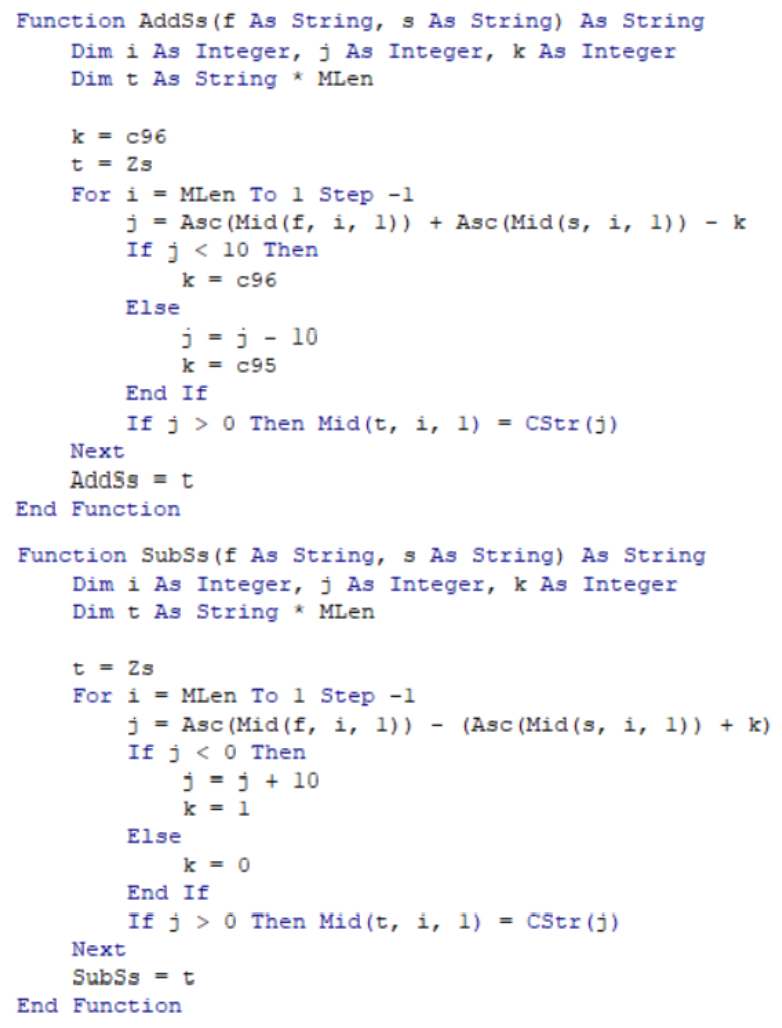



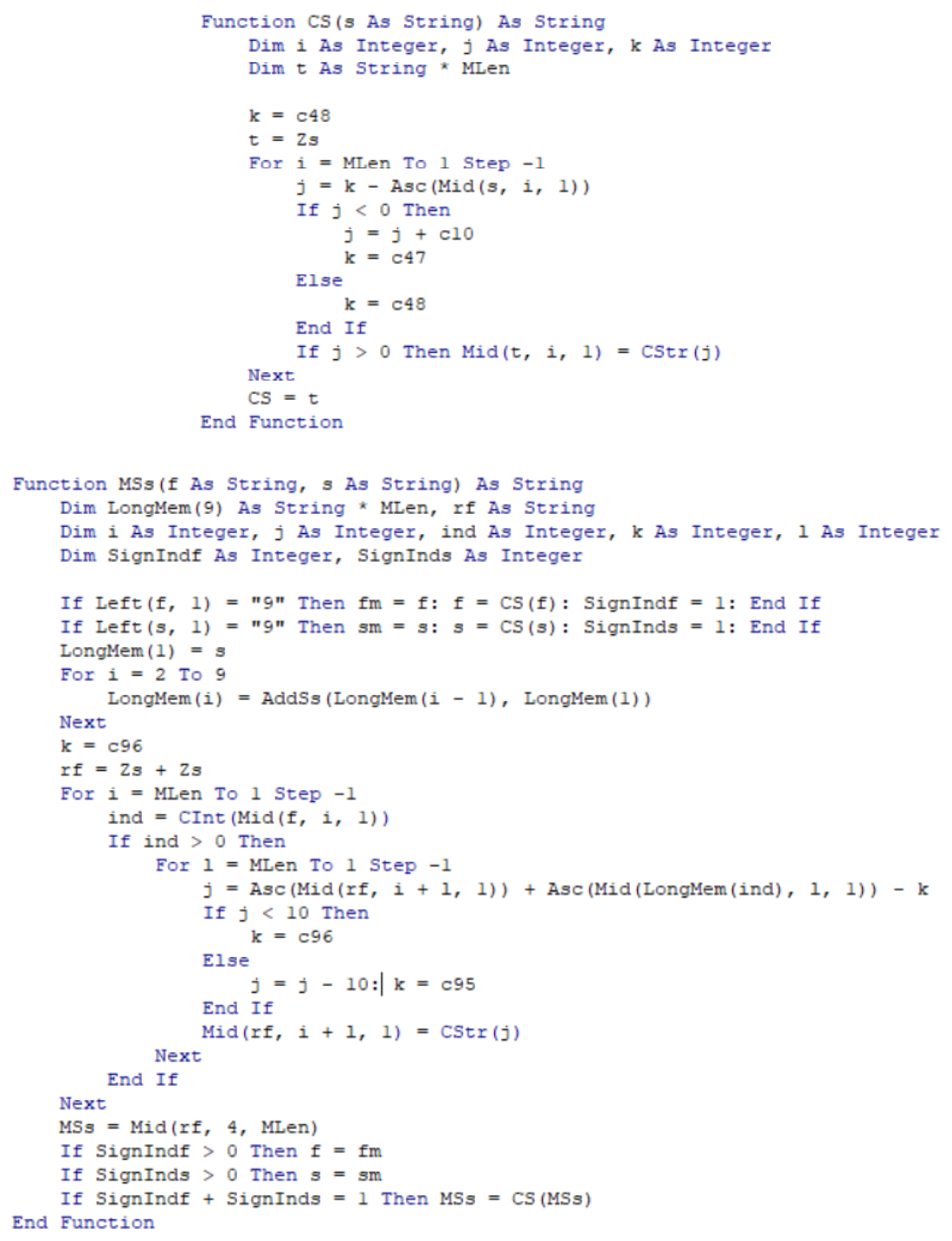


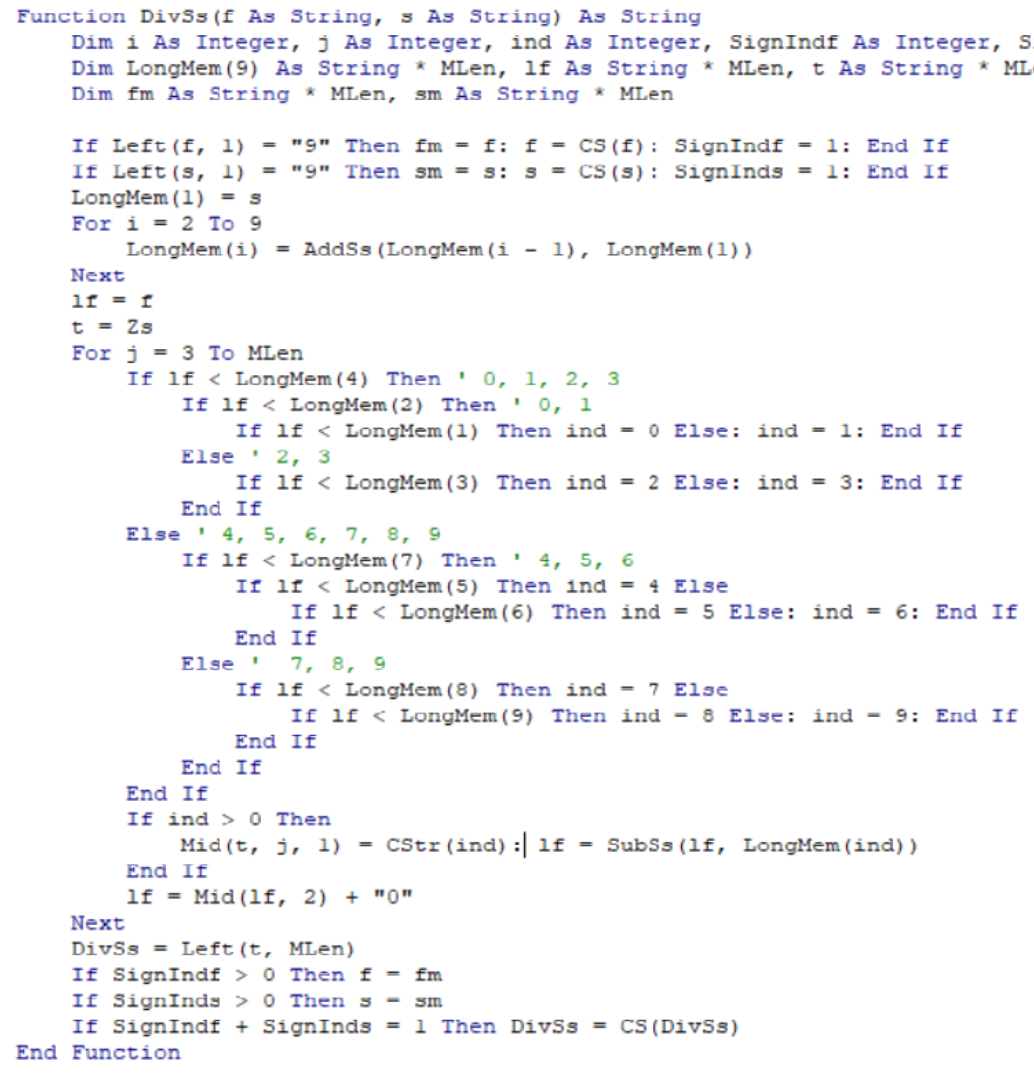

